\title{
Comparing $\mathrm{AM} 200^{\circledR}$ with Titanium-Based Coatings Elastic-Plastic Properties by Nanoindentation, Modified Dimensional Analysis and Minimum Resultant Error Method
}

\author{
Ehsan Bazzaz ${ }^{1} \cdot$ Abolfazl Darvizeh $^{2} \cdot$ Majid Alitavoli $^{2} \cdot$ Mehdi Yarmohammad Tooski $^{3}$
}

Received: 27 April 2019 / Accepted: 10 November 2019 / Published online: 28 June 2020

(c) The Author(s) 2020

\begin{abstract}
Hybrid methods which combine the experimental results with FEM simulations are the most applicable methods to extract the plastic properties of thin-film coatings. In order to make such methods more applicable, they should be merged with the dimensional analysis approach. To make such solution more applicable, a method to reduce the number of dimensionless functions is proposed in order to consider the effect of the probable errors of each parameter. Due to the lack of proper criteria for determining the compliance of the analytical results with empirical data, it was necessary to provide an effective criterion. A complementary new algorithm named as Minimum Resultant Error Method (MREM) is also introduced, which combines the errors of both dimensionless functions. By this algorithm, the yield stress, strain hardening exponent and consequently the strain hardening coefficient are extracted in the form of unique measures. This approach is implemented by minimum empirical data obtained by a single indenter nanoindentation test results. The FEA simulation results are processed with the combination of the aforementioned modified dimensional analysis and MREM algorithms. Such procedure is undergone to calculate plastic properties of AM200 ${ }^{\circledR}$, TiN and TiAlN thin-film coatings. Some of the results, especially for AM200 ${ }^{\circledR}$ coating, are developed for the first time. The results obtained by nonlinear solution of the modified dimensional analysis equations and MREM algorithm are compared with one another. The results of both approaches show very small difference to each other which approves that MREM approach is an effective replacement for nonlinear solution. The sensibility of the answer to the hardening exponent and the errors combining exponent are investigated.
\end{abstract}

Keywords Nanoindentation $\cdot$ Hybrid method $\cdot$ Modified dimensional analysis $\cdot$ Minimum resultant error algorithm $\cdot$ Thinfilm coatings $\cdot$ Titanium-based coatings properties

\section{Introduction}

Titanium-based coatings are broadly used in industrial applications such as machine tools, aerospace, semiconductor technologies as well as some medical applications (Fang et al. 2004; Sun et al. 2015). Specifically, TiN and TiAlN coatings are among those have the widest application in

Abolfazl Darvizeh

adarvizeh@guilan.ac.ir

1 Department of Mechanical Engineering, University Campus 2, University of Guilan, Rasht, Iran

2 Department of Mechanical Engineering, University of Guilan, P.O.B. 41668-13949, Rasht, Iran

3 Department of Mechanical Engineering, Islamic Azad University South Tehran Branch, Tehran, Iran cutting tools for the sake of their excellent characteristics such as high hardness and Young's modulus, low friction (dos Santos et al. 2007), good corrosion resistance and high thermal conductivity (Piscanec et al. 2004). Recently, a new titanium-based type of machine tools coating with the registered mark AM $200^{\circledR}$ has been presented. It is mentioned that its performance and properties are comparable with other titanium-based coatings (Allied Machine \& Engineering Co. 2018).

The main challenge in the coating applications was to create the exactly predefined mechanical properties which represent their actual performance. Difficulties in measuring the mechanical properties of thin films by classical methods have led to the use of indentation method in nanoscale. It has been widely used as a non-destructive test to estimate the material's elastic-plastic properties such as Young's modulus, yield stress, strain hardening coefficient (Patel and 
Kalidindi 2016) and strain hardening exponent (Karimzadeh et al. 2014). Although nanoindentation method has attracted considerable attention in recent decades, extracting the material properties by instrument measured data had serious limitations emerged by difficulties appeared in effectively interpreting the results (Cheng and Cheng 1999). In this regard, extensive analytical and computational studies have been carried out to explain the contact mechanics and the shape deformation during the nanoindentation process (Oliver and Pharr 1992; Giannakopoulos et al. 1994). The main purpose of these studies was to extract the mechanical properties of the materials from the penetration force-depth curve (Dao et al. 2001; Giannakopoulos and Suresh 1999; Suresh et al. 1997; Cheng and Cheng 2004). For instance, the hardness and modulus of elasticity can be obtained from the maximum loading force and the slope of unloading curve, respectively, by the contribution of the proposed method of Oliver and Pharr (1992) and Doerner and Nix (1986).

After the early development of the nanoindentation processing during 1990s, some methods have been devised to derive plastic properties. The foundation of all was relied on comparing the results of finite element simulation with the results of empirical tests. However, the elastic and plastic properties can be calculated using the procedure proposed by Giannakopoulos and Suresh (1999); the residual stress can be obtained by Suresh and Giannakopoulos method (1997). Dao et al. (2001) have succeeded to provide an analytical model that could be utilized to establish a relationship between mechanical properties and the results of nanoindentation test. This method was fundamentally based on some experimental relations that make it too sensitive and difficult to converge. Furthermore, the empirical relations can be used to characterize the properties of specific materials when the under-study ones are exactly fit with the reference one. For the case of the coating materials, the composition and structure are not exactly defined and the size is not big enough to allow testing by the conventional methods. Thus, verification of the empirical relations with the real case is pretty difficult in the majority of cases and impossible yet for the rest. In consequence, Stauss et al. (2003) have used the reverse analysis method to obtain the elastic-plastic behavior of thin films, coatings and microelectromechanical devices. By the same approach, Hong et al. (2016) used the same combination of nanoindentation results and an analytical material behavior model to obtain the power law equation parameters. As the basic difficulties of resolving this problem still remained, attempts have been continued by many researchers for a decade. The latest efforts are paid by Gupta et al. (2015) and Porwal et al. (2016) to resolve the problem with the contribution of sharp indenters test results.

Extensive investigations on the numerical methods have proved that the most appropriate and effective method is the dimensional analysis. The studies of Cheng and Cheng
$(1999,2004)$ are among the first ones succeeded in implementing the dimensional analysis method to extract the dimensionless parameters characterizing the nanoindentation process. One of the most important challenges in this case is the incompatibility of the finite element results with experimental results, especially in terms of the shape of the force-penetration curve. A number of researchers have tried to overcome this problem by creating a variety of test conditions such as changes in the type of indenter. Among others, Heinrich et al. (2009) and Ma et al. (2012) have succeeded in developing a reverse algorithm based on FEM simulation for sharp indenters to acquire the mechanical properties. Consequently, this algorithm has been used by a lot of researchers such as Antunes et al. (2007) and Lee et al. (2009) with some simplifications and further attempts to reach unique answers. Lin et al. (2008) applied the same combination of nanoindentation results and FEM to extract the coefficients of a bilinear equation by using conical indenter. It should be pointed out that most of the above-mentioned methods are established on the basis of iteration algorithms, and then used a large number of dimensionless parameters to reach a certain answer. Some difficulties have been arisen from the experimental and numerical inaccuracies (Antunes et al. 2007), while the others had stemmed from non-uniqueness of the procedure (Challacoop et al. 2003).

The mechanical properties of TiN and TiAlN coatings have been investigated by a large number of researchers (Bazzaz et al. 2019a), whereas AM200 ${ }^{\circledR}$ coating has no published background. In this paper, the mechanical properties of $\mathrm{AM} 200^{\circledR}$ are scrutinized and compared to other most used coatings in cutting tools such as titanium-based coatings. For this purpose, a new approach is proposed to extract the material plastic properties of thin films. Computation algorithm has been developed in such a way that unique values of material properties can be achieved by performing an acceptable amount of calculations. A hybrid method has been developed which combines the results of experimental data with the output of FEM simulation. For different materials, the FEA solution will be adapted with the output of the empirical nanoindentation test to assure that the obtained material properties from the new method are related to the specific material under test. This is the inherent dependency of the method to the type of material. Some simplified conditions have been defined on the conventional dimensional analysis to form a modified dimensional analysis method (MDAM) so that the calculations are considerably reduced. Besides, a novel method named as Minimum Resultant Error Method (MREM) is proposed based on the minimization of a new defined combination of errors. The methodology for both algorithms has been proposed for one indentation curve acquired by one indenter type; therefore, the results are compared with each other for the new case. Actually, the least data for this purpose have been taken into account in order 
to keep the indentation test data and numerical calculation as simple as possible (Wang et al. 2010). Several assumptions in simulating and minimizing the resultant error are applied and discussed, such as the sensibility of the method to, $p$ exponent, variation of Young's modulus and comparison of calculated and measured hardness. All of these studies are done on the results of the Minimum Resultant Error for the first time. The application of this method has already been verified for bulk materials by the authors (Bazzaz et al. 2019b), but the new conditions emerged in thin-film coatings application require a new approach which will be presented here in more details.

\section{Modified Dimensional Analysis Method}

Determining the dimensionless parameters that combine all parameters was the main aim of the conventional dimensional analysis method. From different aspects of dimensional analysis approach, only one parameter is defined for loading course of nanoindentation, and the other dimensionless parameter(s) is defined for both unloading and loading courses. The reason for that is the total number of unknown parameters which are considered at least three parameters (Dao et al. 2001) including elastic modulus in most cases (Fischer-Cripps 2011; Cheng and Cheng 2004). For different types of indenters, more than 3 parameters were usually defined (Heinrich et al. 2009). In the modified (simplified) aspect, the modulus of elasticity can be considered as a given parameter because it has been approved that this parameter can be calculated directly by a straightforward manner with high accuracy from the slope of the unloading force-depth curve (Oliver and Pharr 1992). Therefore, the remained two unknown parameters can be obtained by only two Buckingham- $\Pi$ functions: one can be attained from the loading curve and the other can be taken from both the loading and the unloading curves.

Such functions are almost obtained as the same for different constitutive equations. The constitutive equation is considered in the typical power law form (Fischer-Cripps 2011), which is in good agreement with the actual $\sigma-\varepsilon$ curves. As it was accepted by the majority of researchers, the power law formulation is successfully used to investigate the elastoplastic behavior of soft thin films on hard substrate, metallic thin films, hard thin films on soft substrate and elastic-plastic film on an elastic-plastic substrate (Gupta et al. 2015). The choice of the power law model was supported by two facts. The first one was that it was much more effective than the bilinear model reported in literature. The second one was that in terms of effective prediction it was also superior than the same bilinear model that has been reported in the literature (Porwal et al. 2016). The general form of the power law constitutive equation can be featured out by a nonlinear stress-strain model as the following equation:

$\begin{cases}\sigma=E \varepsilon & \text { For } \sigma \leq \sigma_{y} \\ \sigma=B \varepsilon^{n} & \text { For } \sigma \geq \sigma_{y}\end{cases}$

where $\sigma$ and $\varepsilon$ are stress and strain components, $E$ is the elastic modulus, $n$ is the power law exponent, $\sigma_{y}$ is the yield stress and $B$ is the strain hardening coefficient. To analyze the nanoindentation process, the above equation is used in FEM software such as ABAQUS, to numerically reproduce the indentation force-depth curve. The basis of the dimensional analysis is the output results of nanoindentation test which are displayed as a $P-h$ curve. This curve displays the dependence of the following parameters:

$P=f(B, E, n, h, v, \alpha)$

In which $P$ is the penetration force, $f$ is the function that defines the dependency on the other parameters, $h$ is the penetration depth, $v$ is Poisson's ratio, and $\alpha$ is the indenter cone angle. In the present case, only single indenter results are used. Thus, the last two parameters are constant values and have no effect on dimensionless functions definition. According to dimensional analysis rules and Buckingham- $\Pi$ theory, the dimensionless functions $\Pi_{1}=E^{a} h^{b} P$ and $\Pi_{2}=E^{c} h^{d} B$ relating all parameters to each other can be assumed, where $a, b, c$, and $d$ are necessary exponents to adapt the dimensions of all parameters to each other. By implementing the rules of the dimensional analysis method, the following relations can be achieved:

$\left\{\begin{array}{l}\Pi_{1}=\frac{P}{E h^{2}} \\ \Pi_{2}=\frac{B}{E}\end{array}\right.$

The relation between above two dimensionless functions can be presented as: $\Pi_{1}=f_{1}\left(\Pi_{2}, n, \nu, \alpha\right)$, and the final relationship will become:

$\Pi_{\alpha}\left(\frac{B}{E}, n, v, \theta\right)=\frac{P}{E h^{2}}=\frac{C}{E}$

The relationship between $F$ and $h$ defines the final form of the above dimensionless function. Different researchers used vast types of indenters to illustrate this relationship and concluded the applicability of most of indenters to characterize the material properties. Giannakopoulos et al. (1994) and Antunes et al. (2006) have used Vikers indenter, Dao et al. (2001), Lin et al. (2008), Hong et al. (2016) and many others used sharp indentations by generally conical shape indenters. Suresh et al. (1997) are among the first researchers who have studied spherical indenters, whereas Cao and $\mathrm{Lu}$ (2004) have extended Dao et al. methodology to spherical indenters. The correspondence of the experimental data to FEM results is a decisive factor to choose the indenter type. For conical 
indenters where the parabolic relation is applicable, the method is more compatible with the assumption that is used in developing the dimensionless functions in dimensional analysis method. Kick's law: $F=C h^{2}$ is the commonly used representation form of nanoindentation curves that was used by former researchers such as Dao et al. (2001) and Cheng and Cheng (2004) and the later ones (Hong et al. 2016; Lee et al. 2009). According to Kick's law, $C$ is the coefficient of the estimated parabolic function of the indentation curve: $P=C h^{2}$, which can be calculated by best fit of nanoindentation curves via any conventional numerical method, e.g., the least square method (Giannakopoulos et al. 1994; Dao et al. 2001; Zhaohui and Suresh 2003). When the data raised from the experimental test are compared with the perfect parabolic curve, the error of each point is: $e_{P}=P_{i}-C h_{i}^{2}$ and the total sum of square values of the error $e_{P}^{2}$ for all the data in loading phase is: $S_{r}=\sum_{i=1}^{n}\left(P_{i}-C h_{i}^{2}\right)^{2}$. Minimizing the above square errors sum with respect to $C$ results in the condition: $\mathrm{d} S_{r} / \mathrm{d} C=0$. Such minimization yields $\mathrm{C}$ coefficient as:

$C=\frac{\sum_{i=1}^{n} P_{i} h_{i}^{2}}{\sum_{i=1}^{n} h_{i}^{4}}$

After obtaining the first dimensionless parameter, the other parameter will be derived by considering the unloading curve. In the same way, as done before, a similar function for unloading curve can be developed. New function contains the maximum penetration depth, $h_{\max }$ as a new parameter which defines the beginning of the indentation curve in unloading course. Therefore, the dependence of the parameters is defined as: $P=f\left(B, E, h, h_{\max }, n, \nu, \alpha\right)$. When the dimensional analysis rules are implemented on the above parameters, a new function $\Pi_{3}=E^{g} h^{i} h_{\max }$ can be defined, in which $g$ and $i$ are the necessary exponents to reproduce the new dimensionless parameter. These exponents are calculated so that the final form of above equation becomes: $\Pi_{3}=h / h_{\max }$. Thus, the final relationship is written in the following form:

$\Pi_{\beta}\left(\frac{B}{E}, \frac{h}{h_{\max }}, n, v, \theta\right)=\frac{P}{E h^{2}}=\frac{C}{E}$

So, according to dimensional analysis rules, it can be seen that the above relation between dimensionless parameters can be reversed. By considering the boundary condition: $h=h_{r}$ at $P=0$ in the end of unloading curve (Cheng and Cheng 1999), the last equation becomes:

$\Pi_{\delta}\left(\frac{B}{E}, n, \nu, \theta\right)=\frac{h_{r}}{h_{\max }}$

The analysis can be arranged by considering only two dimensionless parameters $\Pi_{\alpha}$ and $\Pi_{\delta}$ which are dependent on B/E and $n$ only. Also, the calculation of the total energy (in loading course) and plastic energy (considering loading and unloading courses) shows that a linear relationship holds between $h_{r} / h_{\max }$ and $W_{P} / W_{T}$ (Fischer-Cripps 2011) as have been shown for the first time by Loubet et al. in 1984 (Cheng and Cheng 2004). Besides, these two parameters can even be equal in general cases (Dao et al. 2001); hence, the last dimensionless relation can be rewritten as:

$\Pi_{\omega}\left(\frac{B}{E}, n, \nu, \theta\right)=\frac{W_{T}-W_{U}}{W_{T}}=\frac{W_{P}}{W_{T}}$

In which $W_{T}, W_{U}$, and $W_{P}$ are the energies of the loading phase, unloading phase and plastic deformation, respectively (Fig. 1). These parameters are obtained by numerical integration of the instant load, $P$, versus instant penetration, $h$, as follows:

$W_{T}=\int_{\text {Loading }} P(h) d h, W_{U}=\int_{\text {Unloading }} P(h) d h$,

Since solving nanoindentation problems in the classical way requires a lot of iterations and extensive amount of calculations, the conventional method can be replaced by a modified dimensional analysis method (MDAM) on the basis of only two dimensionless parameters. For this purpose, the following set of equations should be solved simultaneously:

$$
\left\{\begin{array}{l}
\frac{C}{E}=\left(\frac{C}{E}\right)^{\exp .} \\
\Pi_{\alpha}\left(\frac{B}{E}, n, \nu, \theta\right)=\frac{C}{E}
\end{array}\right.
$$

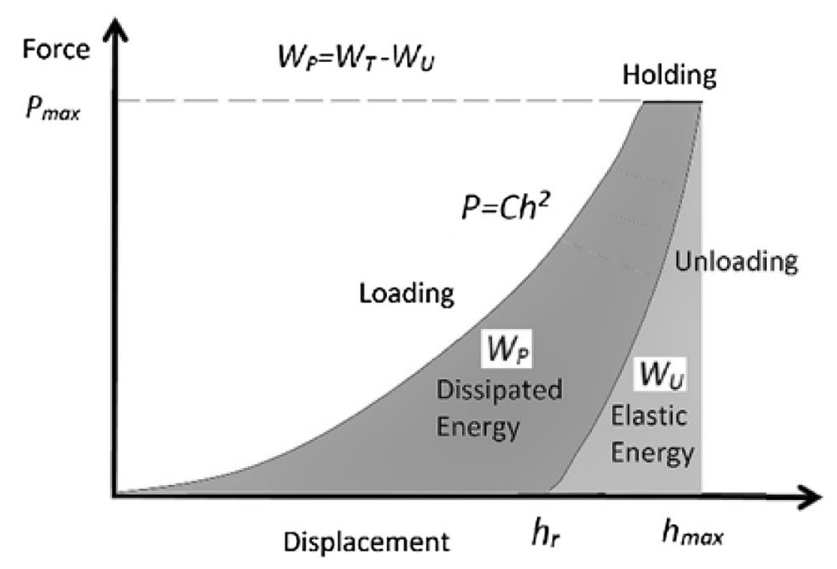

Fig. 1 Force-penetration curves obtained by nanoindentation corresponding with loading and unloading phases with creep effect. The energies are shown as swept areas under curves 


$$
\left\{\begin{array}{c}
W_{P} / W_{T}=\left(W_{P} / W_{T}\right)^{\text {exp }} . \\
\Pi_{\omega}\left(\frac{B}{E}, n, \nu, \theta\right)=\frac{W_{P}}{W_{T}}
\end{array}\right.
$$

For each certain value of $B$, a relevant value of $\mathrm{n}$ can be found so that it satisfies each of the above set of equations separately. But, these two $n$ values are rarely the same for both above equation sets. Fundamentally, the common pair of answers $(B, n)$ between the groups of answers of the two above sets is the unique solution. This answer must achieve the requirements of the following equation system:

$$
\left\{\begin{array}{l}
\frac{C}{E}=\left(\frac{C}{E}\right)^{\exp .}, W_{P /} W_{T}=\left(W_{P} / W_{T}\right)^{\text {exp. }} \\
\Pi_{\alpha}\left(\frac{B}{E}, n, v, \theta\right)=\frac{C}{E} \\
\Pi_{\omega}\left(\frac{B}{E}, n, \nu, \theta\right)=\frac{W_{P}}{W_{T}}
\end{array}\right.
$$

Since the curves created by the results of FEM analysis do not fit with the experimental curves in the majority of cases, two sets of equation systems (10) and (11) usually do not have common answer. If a correction equal to the average of errors in respect to experimental data is considered, the intersection will be reached in most cases. Although the amount of the error for each dimensionless parameter can be estimated by evaluation of the analysis results, its limit is still a big challenge for the analyzers. So, calculations based on dimensional analysis method in both ways: iteration-based and modified methods (MDAM), are very sensitive to any small errors appeared in experiments and data processing (Dao et al. 2001; Cheng and Cheng 2004). Considering this, in spite of the fact that MDAM algorithm reduces the calculations considerably, it is still not an easy way to predict the main material properties. In order to overcome such difficulties, an effective procedure is required to calculate the constitutive equation constants. Therefore, a new method on the basis of minimum error emerged with a hybrid method dealing with the fitment of FEM results to experimental data, is developed. Due to the combination of the errors of both parameters, a special criterion is assumed to evaluate the errors. First, the errors in reference to experimental results are defined:

$$
\begin{gathered}
e(C / E)=\frac{C_{\exp } / E-C_{\text {num }} / E}{C_{\text {exp }} / E} \\
e\left(W_{P} / W_{T}\right)=\frac{{\frac{W_{P}}{W_{T}}}^{\exp }-\frac{W_{P}{ }^{\text {num }}}{\frac{W}{T}_{P}^{\exp }}}{W_{T}}
\end{gathered}
$$

where $e(C / E)$ is the error of $\Pi_{\alpha}$ and $e\left(W_{P} / W_{T}\right)$ is the error of $\Pi_{\omega}$ functions. So, the subscripts and superscripts, exp and num denote the experimental and numerical results, respectively. As a basis to the combination of the errors, Wang et al. (2010) have used the summation of the absolute values of all errors. This assumption seems to be a basic necessity, since the sign of the errors is not as important as the magnitude of them. As this condition is challenged by the results of many analytical cases, it seems that it is not a sufficient criterion to cover all cases. That is because it gives all errors an equivalent role in the total error. Instead of it, a proper criterion has to achieve this condition: to give more role to $e(C / E)$ than $e\left(W_{P} / W_{T}\right)$. To fulfil such assumption, a metric space sum of the errors is proposed:

$e_{\text {rte }}=\left[|e(C / E)|^{p}+\left|e\left(W_{P} / W_{T}\right)\right|^{p}\right]^{1 / p}$

The parameter $e_{\text {rte }}$ is defined as the resultant total error. The exponent $p$ is a natural number indicating the rank of metric combination, which can be determined through the minimization procedure of $e_{\mathrm{rte}}$. The resultant error function is used as a unique criterion to evaluate the fitment of the FEM results to experimental data. The final unique set of the mechanical properties is relevant to the minimum value of above resultant total error, among all minimums obtained for each $(B, n)$ set. This value is the infimum value of the combined errors and can be defined as:

$(B, n)=\left\{(B, n) \mid e_{\mathrm{rte}}=\operatorname{Inf}\left(e_{\mathrm{rte}}(B, n)\right)\right\}$

where the $\operatorname{Inf}\left(e_{\text {rte }}(B, n)\right)$ is defined as: $\operatorname{Min}\left(\operatorname{Min}\left(e_{\mathrm{rte}}(n) \mid B=\right.\right.$ cte. $\left.)\right)$. This procedure which is used in all over this paper is mentioned as MREM (Minimum Resultant Error Method). The new methodology is used to determine the material properties for all thin films even their specification and chemical composition are not well known. It should be pointed out that more than one minimum may be obtained over the entire parameters domains, which called local minima. Energy rules for initiation of plastic behavior state that the case with minimum energy level is the one that starts the plastic state. The procedure to implement the above approach is shown in the flowchart of Fig. 2.

\section{Nanoindentation Test}

The samples are selected of spade drill tools manufactured by Allied Machine and Engineering Company (AMEC) (2018) as shown in Fig. 3. All coatings are deposited by CVD method on substrate of cobalt superalloy high-speed steel: M42. TiN and TiAlN coatings are well known and commonly used coatings, but AM200 ${ }^{\circledR}$, which is rarely studied before, is an exclusive trade mark product belonging to AMEC. The coated cutting tools have undergone a nanoindentation test to obtain the $P-h$ curve which is used to extract the material properties of thin films. The 


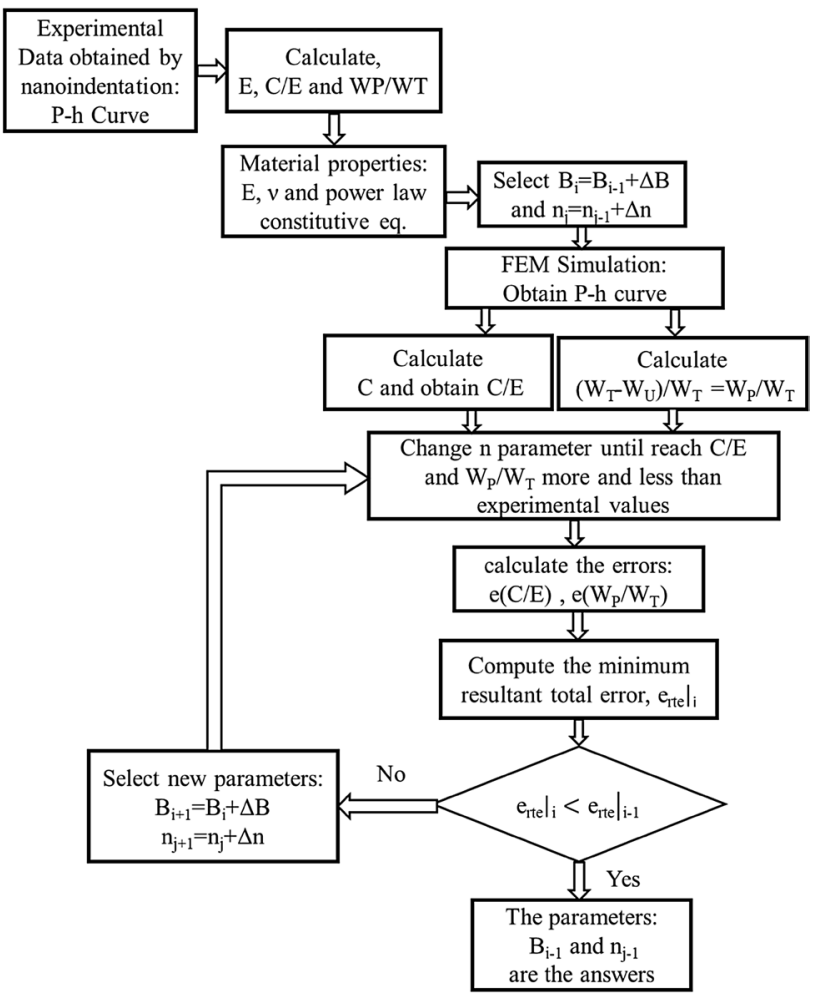

Fig. 2 Flowchart of reverse algorithm based on modified dimensional analysis (MDAM) and minimum resultant error (MREM) methods

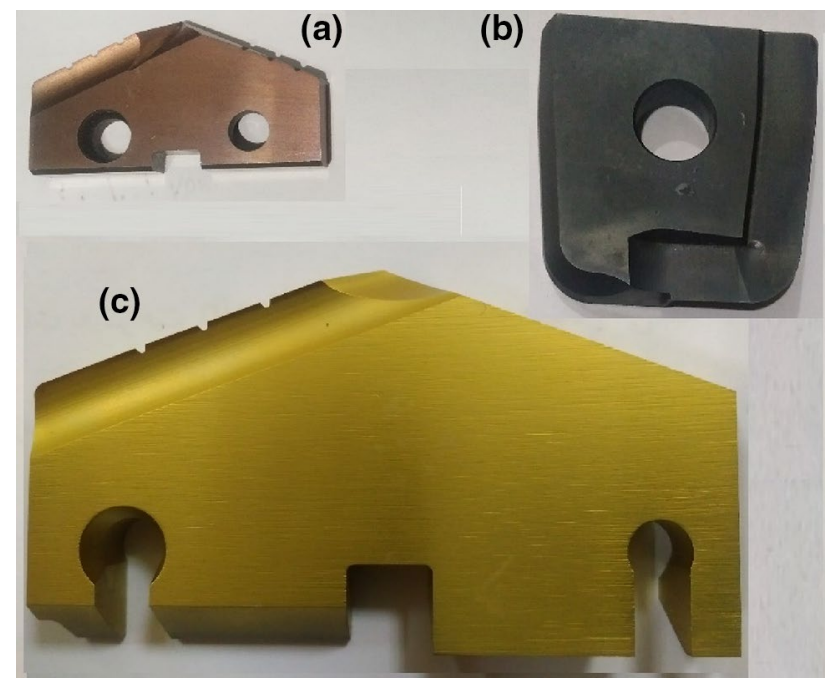

Fig. 3 Three samples of Spade drill blades coated by: a AM200 ${ }^{\circledR}$, b TiAlN and c) TiN

nanoindentation test for all samples was carried out by an Anton Paar nanoindenter (NHT3 Type) working with depth sensing instruments and using Berkovich indenter. The samples surfaces were cleaned and slightly polished to a proper surface quality so that thin-film layer is not harmed. The record resolution for the force and depth is $0.01 \mu \mathrm{N}$ and
$0.01 \mathrm{~nm}$, respectively. Test procedure was conducted according to ISO14577 (2002) and its equivalent ASTM E2546 standard (2003).

As explained in details by the literature, the critical depth ratio (CDR) is an important criterion to evaluate the effect of the substrate on the properties of a thin-film coating. There is a strict dependency of CDR to proportional properties of the coating and substrate. Early studies of Lichinchi et al. (1998) illustrated that CDR ratio should be less than $10-20 \%$ for soft thin films, while it should be less than $10 \%$ for hard thin films. Detailed investigation of Gupta et al. (2016) revealed that when the coating is relatively softer than the substrate, the variation of this ratio is as wide as $10-40 \%$. For the inverse relation, when the coating is relatively harder than substrate, this ratio is changing to $5-20 \%$ range. To specify the applicable range for any specific coating, more investigation is necessary. For AM $200^{\circledR}$ thin film which has material properties close to titanium-based coatings, CDR can be considered as maximum as \%11 (Kataria et al. 2012). One of the preferred ways to assess the effect of the substrate on the thin films is the study of the variation of the elastic modulus as a function of the indentation depth to film thickness ratio. For this purpose, the data obtained from nanoindentation tests with various penetration depths for $\mathrm{AM} 200^{\circledR}$ are used to report the variation trend of elastic modulus versus penetration depth. Figure 4 a shows that elastic modulus with standard deviation of $3 \%$ has roughly the same values up to the ratio of $11 \%$ of penetration depth to film thickness. This result supports the conclusion made by Kataria et al. (2012) that the nanoindentation results of TiN thin films with penetration ratio lower than $11 \%$ are not affected by substrate.

Indentation force was adjusted to produce an indentation depth within the minimum acceptable limit of the film thickness (Cheng and Cheng 2004). In proportion to coating strength, the maximum force was set on 20,80 and 120 $\mathrm{mN}$ for TiAlN, AM $200^{\circledR}$ and TiN, respectively. The test is repeated 5 times for each sample. The results of all indentations for $\mathrm{AM} 200^{\circledR}$ coated cutting tool are shown in Fig. 4b. All curves feature out similar trend and limits of the data and have clear overlap, so that the reference indentation $P-h$ curve can be obtained by the average of the in-range data as shown in Fig. 5. The resulted maximum penetration depth has reached 488, 308 and $398 \mathrm{~nm}$ for TiN, TiAlN and AM $200^{\circledR}$ samples, respectively. The most important elastic property, the elastic modulus, is calculated from $P-h$ curves (Oliver and Pharr 1992) as $504.4 \mathrm{GPa}$ for $\mathrm{AM} 200^{\circledR}$, while it was 581 and $272.2 \mathrm{GPa}$ for TiN and TiAlN, respectively. Using the same data, the loading curvature character, C, is calculated via polynomial second-order interpolation as $539 \mathrm{GPa}$. So, by computing the areas falling under the loading and unloading curves, the other dimensionless parameter $W_{P} / W_{T}$ is obtained as 0.5218 . The shape of the indentation 

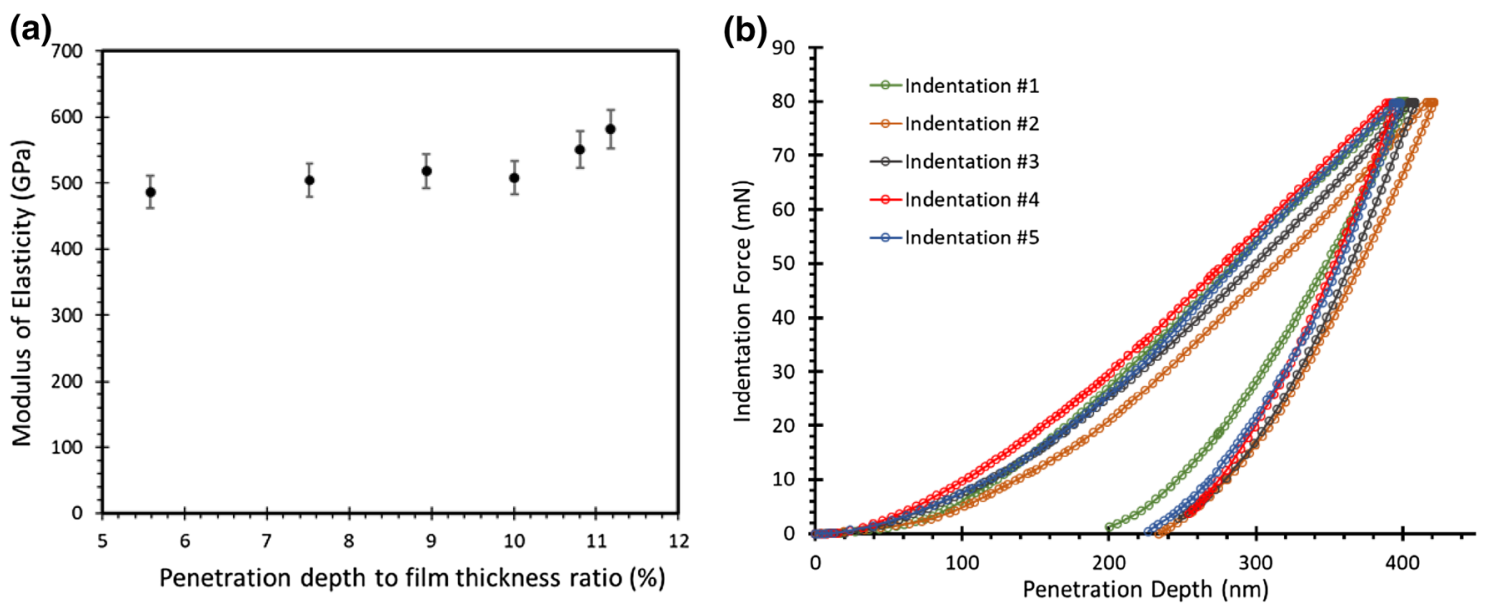

Fig. 4 a Variation of elastic modulus versus the ratio of penetration depth to film thickness with $3 \%$ standard deviation and $\mathbf{b}$ results of 5 indentations for $\mathrm{AM} 200^{\circledR}$ coating on cutting tool steel substrate

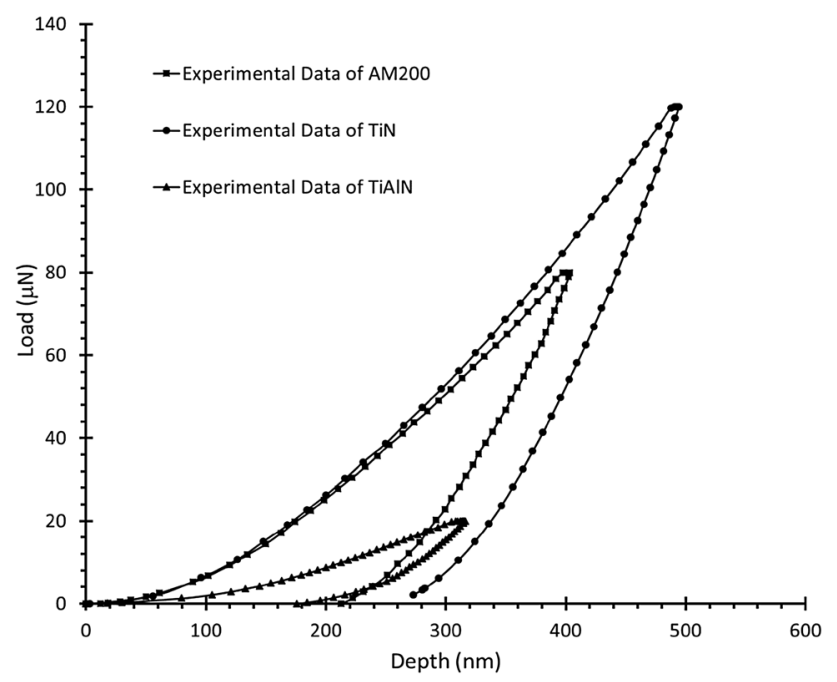

Fig. 5 Experimental results of nanoindentation measurement on TiN, TiAlN (Bazzaz et al. 2019a) and AM200 ${ }^{\circledR}$ thin-film coatings on M42 cutting tool steel

curves revealed that there is no evidence of pop-in phenomenon, i.e., grain boundary slippage and buckling. This fact leads to the conclusion that the results of FEM analysis can replicate the actual behavior of the material. A little evidence of creep behavior is observed in the end of the loading course (i.e., the holding course) which should be removed when the curves are compared with the simulation results.

Photography by SEM has been conducted to distinguish the scale of the metallurgical structure of the coating. To make better appearance on the sample surface and consequently better detection of the material structure, a very thin layer of gold (about $10 \mathrm{~nm}$ ) is deposited by PVD on the sample surface. The photograph of the sample surface in low and best quality regions, far from and near to the cutting edge, is shown in Fig. 6. Although topography of side surfaces displays non-compact structure with considerable porosity, the surface near cutting edges clearly demonstrates a relatively dense and smooth structure without any sign of cracks and pores. Investigations reveal that the mean placket/phase size of $\mathrm{AM} 200^{\circledR}$ is smaller than the size of triangle side of the indentation hole $(\sim 1500 \mathrm{~nm})$. That is why, the tests that have been done under higher indentation forces and consequent deeper penetrations provide more satisfactory results. Another achievement of the photography was to measure the thickness of the coating without need to prepare a section of the sample. By photography in $15^{\circ}$ angle perpendicular to the sample surface, the thickness is calculated in the range of 5-6 $\mu \mathrm{m}$. So, XRD analysis can give valuable information about the material of the thin film especially in AM200 ${ }^{\circledR}$ case when the composition of the coating was not provided. The X-Ray Diffraction (XRD) test is done on the sample by a Philips PW1730 facility with $\mathrm{Cu}$ anode, $1.544 \AA$ A wave length and $30 \mathrm{~mA} / 40 \mathrm{kV}$ generator setting. The results reveal that the coating includes diversity of elements and compounds such as chromium, chromium nitride, titanium, titanium carbide and carbon. The phase diagram in Fig. 7 demonstrates many picks that represent crystalline structure of the coating and show no sign of amorphous phases.

\section{FEA Simulation of Nanoindentation}

Since more realistic simulation assumptions affect the fitment of numerical results to experimental data significantly, careful selection of the simulation parameters plays a key role in the accuracy of the output data. The basic FEM model has been created using commonly wellknown ABAQUS software. The main part of the model is fabricated of the substrate, thin film, and the indenter. 

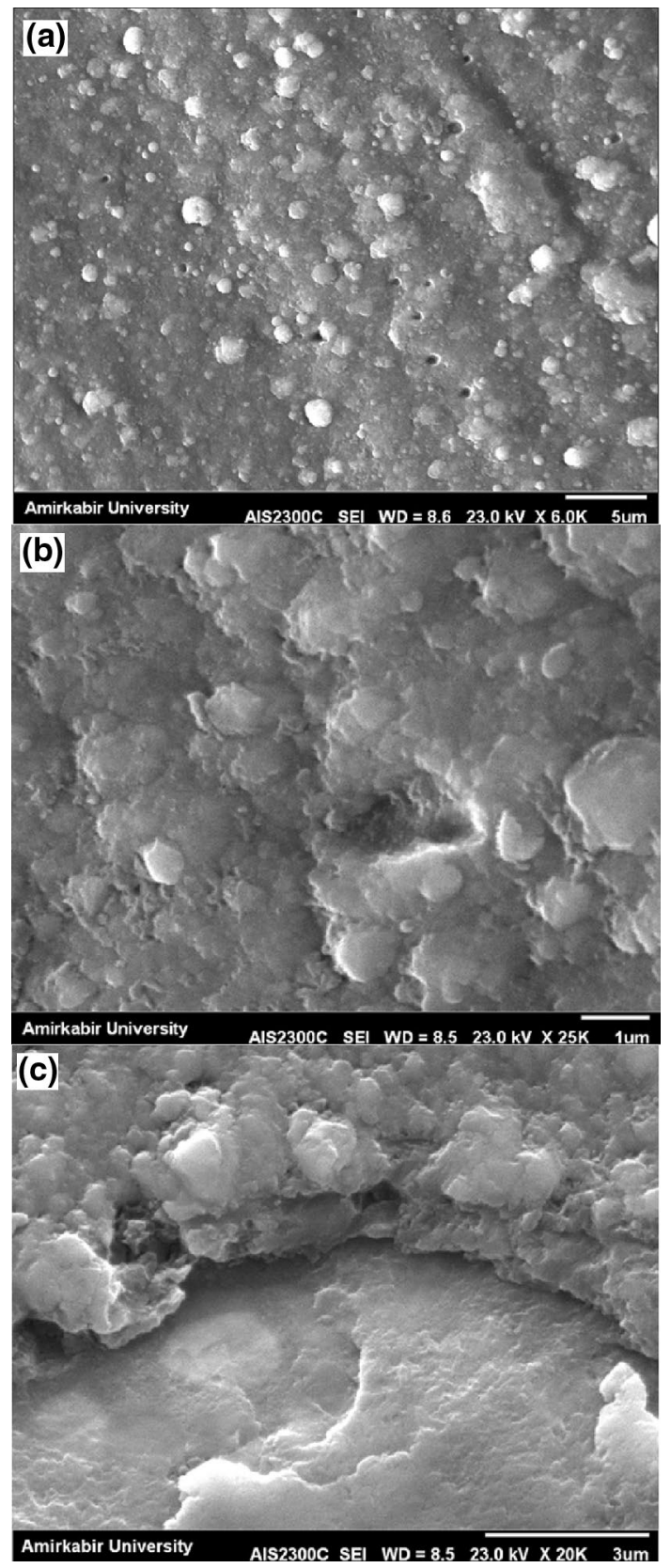

Fig. 6 Topography of $\mathrm{AM} 200^{\circledR}$ coating surface in: a Side of spade drill, $\mathbf{b}$ near the cutting edge, and $\mathbf{c}$ on the cutting edge

The whole model is prepared as a cylinder with a cone that demonstrates the sample and the indenter, respectively. Since all model sections that pass through the axis of the cylinder behave completely similar to each other, the assumption of asymmetry is completely applicable. That's why this condition is assumed by the majority of the researchers (Patel and Kalidindi 2016; Cheng et al. 2002; Bobzin et al. 2007), although 3D models were employed in a few research works (Dao et al. 2001; Kang et al. 2012; Antunes et al. 2006).

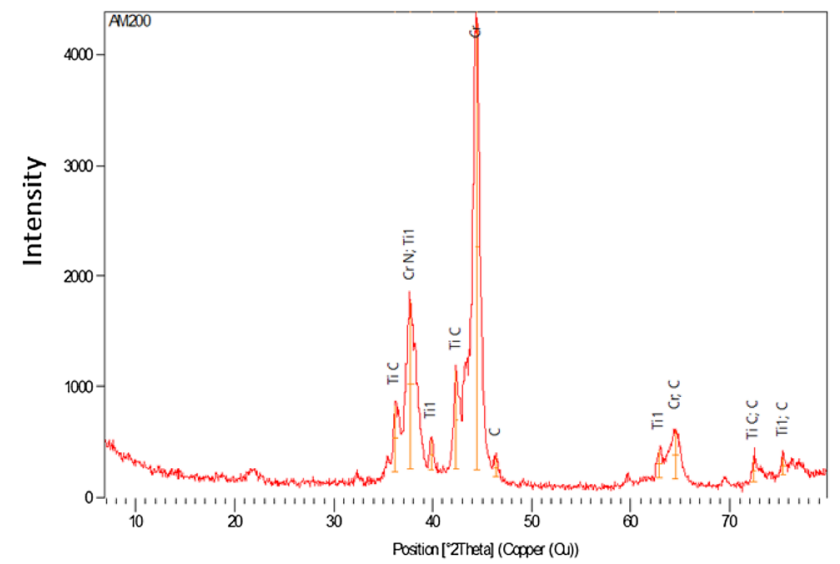

Fig. 7 Spectrum diagram of phase distribution respect to $2 \times$ Theta of X-Ray beam position which show a vast of picks and crystalline phases

The refinement of the mesh plays a key role in the convergence of the results and lowers the simulation errors, especially when the element's minimum size is comparable with the penetration depth. Therefore, the meshing of the material should be refined in such a way that ensures the convergence of the solution. Since the coating thin film has a very little comparative thickness $(5 \mu \mathrm{m})$, there should be enough elements to allow proper demonstration of the material behavior. A combination of triangle and rectangle axisymmetric elements is used to model the layered material so that the size change of the elements can be managed in minimum area. The elements in the vicinity of the indenter tip should be refined more due to the high expected stress gradients. For the present work, a rectangle of $1 \mathrm{~mm} \times 1 \mathrm{~mm}$ is meshed and refined as seen in Fig. 8.

As a reliable assumption, Berkovich indenter model was considered as a perfect cone with semi apical angle of $70.3^{\circ}$ in the axisymmetric model which has the same projected area to depth function as the standard Berkovich indenter (Lichinchi et al. 1998). The indenter is usually defined as a perfectly rigid body, meaning that the deformation of the indenter tip is neglected. Although deformable indenters have been modeled in some studies (Malzbender 2002; Sakharova et al. 2009), there was no evidence of significant difference in the output results when it is compared to the FE models with a rigid indenter (Karimzadeh et al. 2014). From the view of the analysis method, it is completely possible to consider the tip of the indenter as a perfectly sharp cone, but the results are significantly different when the tip radius changes. Extensive numerical calculations for a lot of cases have revealed that a slight tip curvature is necessary for more realistic FEM results. Former studies of the authors indicate that a tip radius close to the penetration depth gives more correspondent results comparing with experimental data (Bazzaz et al. 2019b). 
Fig. 8 Final mesh refinement of the coated sample including indenter penetration zone, thin film, substrate and indenter

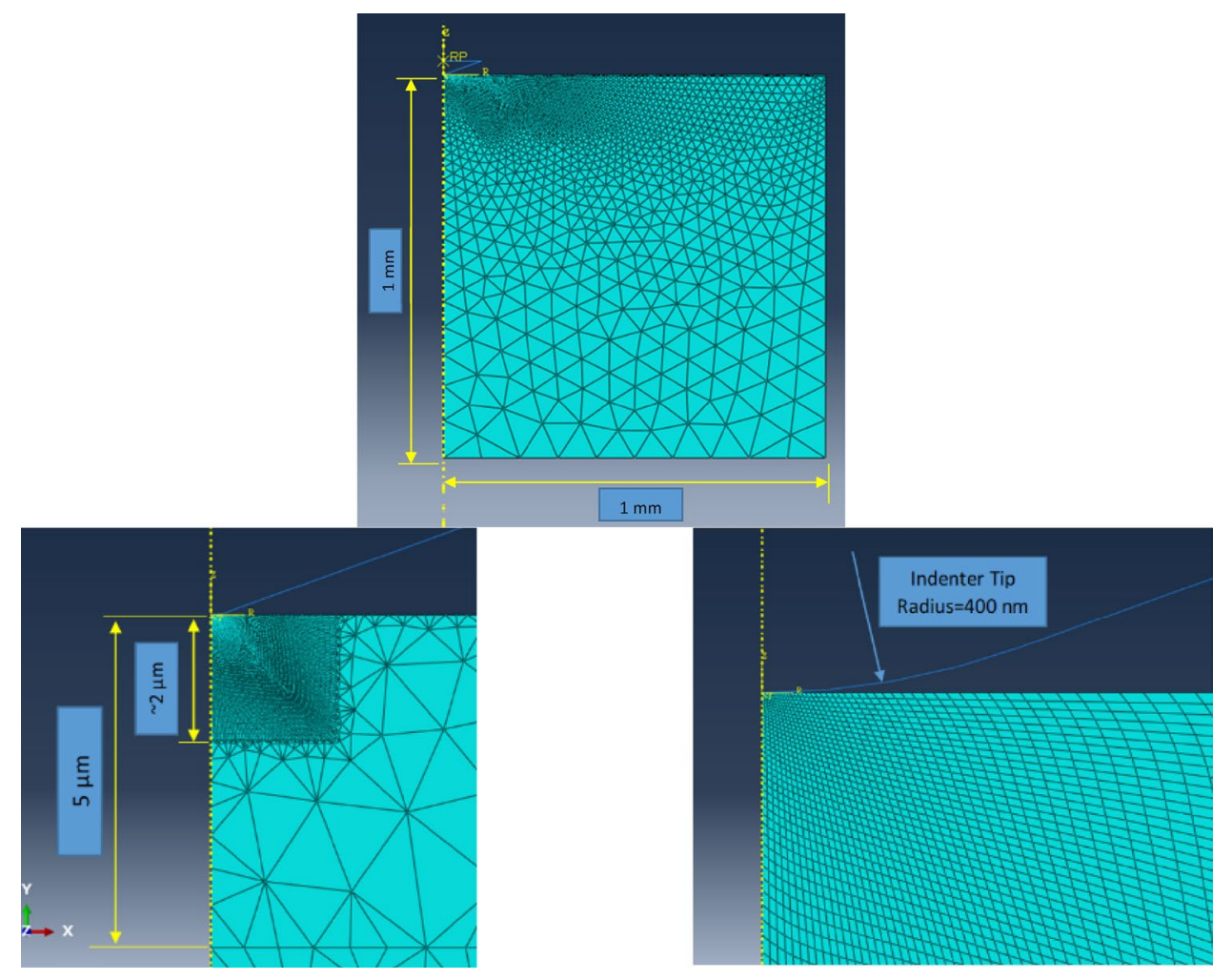

The friction between the indenter and the substrate surface is assumed to be zero by most researchers (Lichinchi et al. 1998; Duodu et al. 2016), especially when the material is pretty hard. Regarding the determination of material properties, which is mostly about obtaining the load-displacement curves, the influence of contact friction during the indentation is limited (Wen et al. 2017). On the other hand, frictionless assumption in ABAQUS solver makes a constraint on the mating surfaces of the indenter and substrate, which prevents them separating from each other when it is necessary. Therefore, the friction between the indenter and the sample surfaces has been taken into account in the majority of the studies (Vaidyanathan et al. 2001; Li et al. 2013). In this study, the friction coefficient is considered as 0.4 , in the average range of variation of most titanium-based coatings.

Contact surfaces are regarded as sliding surfaces constructed by contact elements so that the indenter outside surface is the master and the upper face of the sample is the slave. The contact direction is then defined from the indenter surface toward the sample due to the fact that the master is a rigid body and only its surface can penetrate into the slave body (Lichinchi et al. 1998; ABAQUS 6.14 2014; Duodu et al. 2016).

The boundary condition of the nodes in the bottom surface is usually defined as full constrained along with and perpendicular to the direction of symmetry axis. All nodes on the rotation axis are automatically defined as fixed in the direction perpendicular to the penetration force. The loading and unloading conditions of the indentation process can be applied using load control or displacement control regimes which are both allowed by software options. The displacement control is selected, since it gives direct control over the indentation scale (micro-indentation or nanoindentation). The total indentation depth will be divided into several increments depending on the stability of the analysis. At each depth increment, many iterations are managed by the software according to a specified convergence rate to reach an adequate accuracy (Lichinchi et al. 1998; ABAQUS 6.14 2014; Duodu et al. 2016).

\section{Material Behavior}

The materials of the test samples are assumed to be homogeneous and isotropic all over the present study. The substrate is considered as a full elastic material due to the fact that the maximum stress in the substrate is lower than the yield stress of HSS M42 material. In this case, full elastic assumption does not lead to any different results compared to the elastic perfectly plastic assumption. The chemical composition of HSS M42 is noticed according to the commonly used ASM reference (1989) according to Table 1.

The mechanical properties of $\mathrm{AM} 200^{\circledR}$, TiN and TiAlN thin-film coatings are the required output of the analysis, which are considered as the result of implementing the 
Table 1 Chemical composition of tool steel HSS M42

\begin{tabular}{lllllll}
\hline Carbon & Silicon & Chromium & Vanadium & Tungsten & Molybdenum & Cobalt \\
\hline 1.1 & 0.4 & 3.88 & 1.15 & 1.5 & 9.5 & 8.25 \\
\hline
\end{tabular}

Table 2 Calculation of Young's Modulus for the coating film based on the experimental data with both deformable and rigid indenter assumption

\begin{tabular}{|c|c|c|c|c|c|c|}
\hline Indenter flexibility & Sample identification & $\begin{array}{l}E_{i}(\mathrm{GPa}) \text { indenter } \\
\text { Young's modulus }\end{array}$ & $\begin{array}{l}E_{r}(\mathrm{GPa}) \text { effective } \\
\text { Young's modulus }\end{array}$ & $\begin{array}{l}v \text { coating Pois- } \\
\text { son's ratio }\end{array}$ & $\begin{array}{l}v_{i} \text { indenter } \\
\text { Poisson's ratio }\end{array}$ & $\begin{array}{l}E_{f}(\mathrm{GPa}) \text { coat- } \\
\text { ing Young's } \\
\text { modulus }\end{array}$ \\
\hline Deformable & $\mathrm{AM} 200^{\circledR}$ & 1141 & 363.7 & 0.23 & 0.07 & 504.4 \\
\hline Rigid & AM $200^{\circledR}$ & $\infty$ & 363.7 & 0.23 & 0 & 344.4 \\
\hline Deformable & TiN & 1141 & 402.3 & 0.25 & 0.07 & 581 \\
\hline Rigid & TiN & $\infty$ & 402.3 & 0.25 & 0 & 377 \\
\hline Deformable & TiAlN & 1141 & 227.3 & 0.2 & 0.07 & 272.2 \\
\hline Rigid & TiAlN & $\infty$ & 227.3 & 0.2 & 0 & 218.2 \\
\hline
\end{tabular}

reverse algorithm. For the indenter, when it is assumed as a rigid body, there is no necessity to consider its characteristics in the model, but the mechanical properties of the diamond should be assumed to calculate the equivalent modulus of elasticity. In the elastic zone, the main coatings characteristic is the elastic modulus which can be obtained from the commonly used relation with the following composite formulation:

$E_{r}=\left[\frac{E_{f} E_{i}}{E_{f}\left(1-v_{i}^{2}\right)+E_{i}\left(1-v_{f}^{2}\right)}\right]$

In which $E_{r}$ is the effective modulus of elasticity, while $E_{f}$ and $E_{i}$ are thin film and indenter elastic modulus, respectively. So, $v_{i}$ is the indenter and $v_{f}$ is the thin-film Poisson's ratios. When the indenter material is considered almost rigid, its modulus of elasticity tends to infinity, thus the adapted Young's modulus $E_{f}^{r}$ can be calculated from the following relation:

$E_{f}^{r}=\left(1-v^{2}\right) E_{r}$

According to previous studies on titanium nitride nanoindentation experimental data, where $E_{f}=581 \mathrm{GPa}$ and $v=0.25$, and when the mechanical properties of the diamond, $E_{i}=1141 \mathrm{GPa}$ and $v=0.07$, are assumed for the indenter, the modulus of elasticity for rigid indenter is obtained as $377 \mathrm{GPa}$. Similarly, the calculation is repeated for AM200 ${ }^{\circledR}$ and TiAlN-coated samples. The Poisson's ratio of AM $200^{\circledR}$ is considered 0.23 as the same as the mean values of TiSiN, which probably contains similar elements. Table 2 shows the elastic mechanical properties of above three types of coatings by considering the assumptions of deformable and rigid indenters.

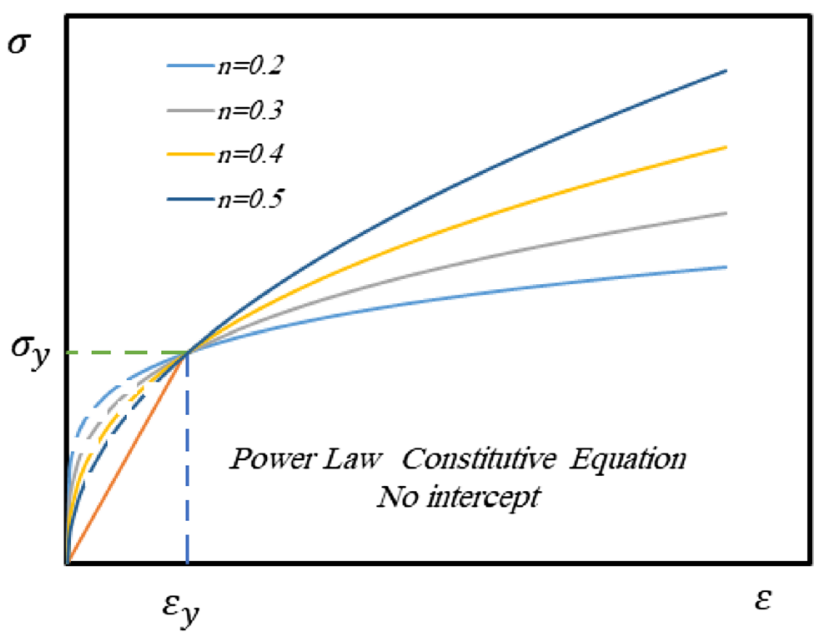

Fig. 9 Schematic representation of the stress-strain diagram for elastic-plastic behavior in the power law general form with zero intercept and the sensibility to strain hardening exponent

For the plastic zone of thin-film coating, the power law constitutive equation is considered in most studies. As mentioned by Gupta et al. (2015) and Porwal et al. (2016), the main reason of this issue is that it is the most applicable rule to demonstrate plastic behavior for most materials, especially metals (Wen et al. 2017). With the assumption of zero interception, as shown in Fig. 9, power law relation can be demonstrated in the form of Eq. (1). In case the general form of Johnson-Cook equation, $\sigma=\left(A+B \varepsilon^{n}\right)\left(1+C \ln \dot{\varepsilon}^{*}\right)\left(1-T^{* m}\right)$ is used to demonstrate the plastic state behavior (ABAQUS 6.14 2014); yield stress should be placed instead of A (Stopel and Skibicki 2016). The other conditions, when strain rate and temperature change are absent, can be specified as: $B \neq 0, C=0, T^{*}=0$. Hence, the final relation is reduced to: 
$\sigma=B\left[\varepsilon^{n}+\left(\frac{\sigma_{y}}{E}\right)^{n}\right]$

\section{The Modified Dimensional Analysis Algorithm}

The MDAM algorithm can be used as a strong mean to achieve the main plastic properties. For the bulk and coated materials, this procedure was successfully examined and frequently applied by the authors (Bazzaz et al. 2019a, b). The plastic characteristics of TiN and TiAlN coatings are obtained from the previous studies of the authors. In this paper, the same procedure is applied to determine the plastic properties of $\mathrm{AM} 200^{\circledR}$ coating and the results are compared with similar data of TiN and TiAlN coatings. At first, FEM simulation should be undergone to obtain the results for full coverage of the parameters domains. Thus, the search is possible to be done for the minimum resultant error. Since $B$ and $\sigma_{y}$ are dependent to each other, $\sigma_{y} / E$ is considered as the main parameter instead of $B / E$.

The entire analysis is carried out with the assumptions of: 2D axisymmetric model, friction coefficient 0.4 , rigid indenter and tip radius $400 \mathrm{~nm}$. Elastic modulus is extracted from the experimental $P-h$ curve and relation (20) as $E=344.4 \mathrm{GPa}$ (for rigid indenter), while the power law constitutive equation parameters are used to simulate the elastic-plastic behavior. Comprehensive investigations illustrate that yield stress is usually below the limit $0.01 \mathrm{E}$ which is a proper start point for the analysis, while the strain hardening exponent is considered from 0 up to 0.5 for most of metals. For other metals, there is an effective way to reach the limits of the yield stress. That is to select the arbitrary yield stress and use FEA simulation so that $P_{\max }$ values are obtained in the domain including experimental $P_{\max }$ value. Yield stresses providing $P-h$ curves totally above or underneath the $P-h$ experimental curve are out of order. This procedure is completely independent from the material type and only depends on the variation trend of the experimental data. The method is more adaptable to materials that generate experimental curves near parabolic shape or the curves follow Kick's law.

As per the reverse method chart in Fig. 2, the calculations are carried out to find the infimum of the resultant error. Following to each simulation run with determinate values of the yield stress and hardening exponent, the individual errors of dimensionless parameters and the resultant errors are calculated. This procedure allows us to search the conditions that generate the absolute minimum resultant error. The calculation is repeated 49 times for the entire probable answer zone. A sample of a set of results, for $\sigma_{y}=4250 \mathrm{MPa}$ and a variation of $\mathrm{n}$ up to 0.5 , are introduced as a series of $P-h$ curves in
Fig. 10. With each set of data, the dimensionless parameters are $\Pi_{\alpha}$ and $\Pi_{\omega}$; thus, the resultant error can be calculated. It is clearly observed that the values of these parameters can be more and less than the experimental measures. That is why, a minimum error compared to experimental data can be achieved.

Repeating the simulation for different $\sigma_{y}$ and $n$ (and consequently $B$ ) results in the variation of dimensionless parameters $\Pi_{\alpha}$ and $\Pi_{\omega}$. A group of curves for the variations of $\Pi_{\alpha}$ and $\Pi_{\omega}$ with respect to plastic characteristics (i.e., yield stress and hardening exponent) are shown in Fig. 11a, b. Although the curves are almost linear in this case, they generally have nonlinear and nonregular form. In general, investigations on the variations of the dimensionless functions show that the minimum amount of both of them is unlikely to be achieved in certain yield stress and hardening exponent, simultaneously. However, the inverse trend of the variation of the parameters implies that a unique value may exist to fulfil the requirement of minimum error for both of them. The simultaneous solution of Eqs. (10) and (12) requires that the intersection of above set of lines with experimental limit be obtained. Such Lucas of intersections performs a curve for each parameter, as drawn in Fig. 12. Eventually, the intersection of aforementioned curves is determined to find the unique answer of the nonlinear set of Eqs. (12). The final result is: $\sigma_{y}=4200$ (MPa), $n=0.47$ and $\mathrm{B}=33,533(\mathrm{MPa})$.

\section{Minimum Resultant Error Algorithm}

The application of MDAM approach brings about effective results when the variations of the dimensionless parameters are almost linear and the fitment of the simulation results to experimental data is almost perfect. To avoid

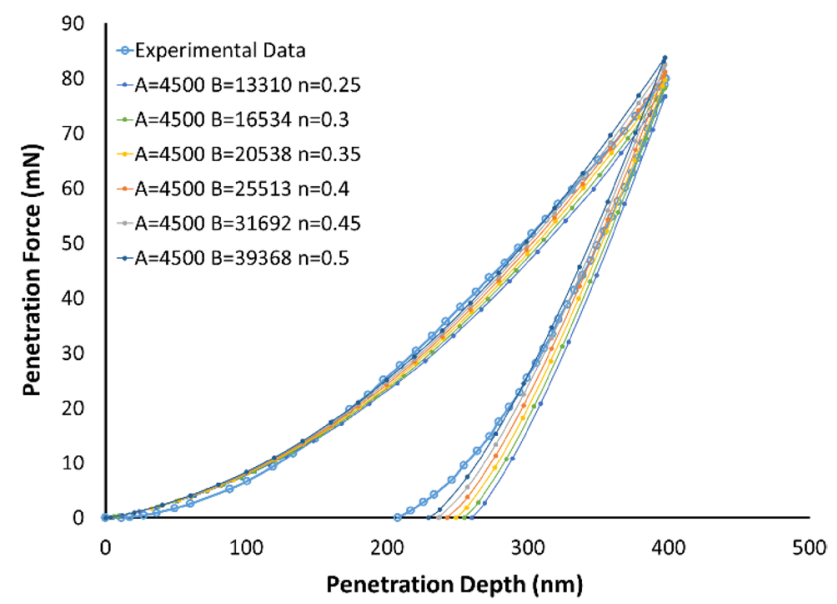

Fig. 10 A series of $P-h$ curves resulted from FEM analysis for AM $200^{\circledR}$ coating with $\sigma_{y}=4500 \mathrm{MPa}$ and $025 \leq n \leq 0.5$ 

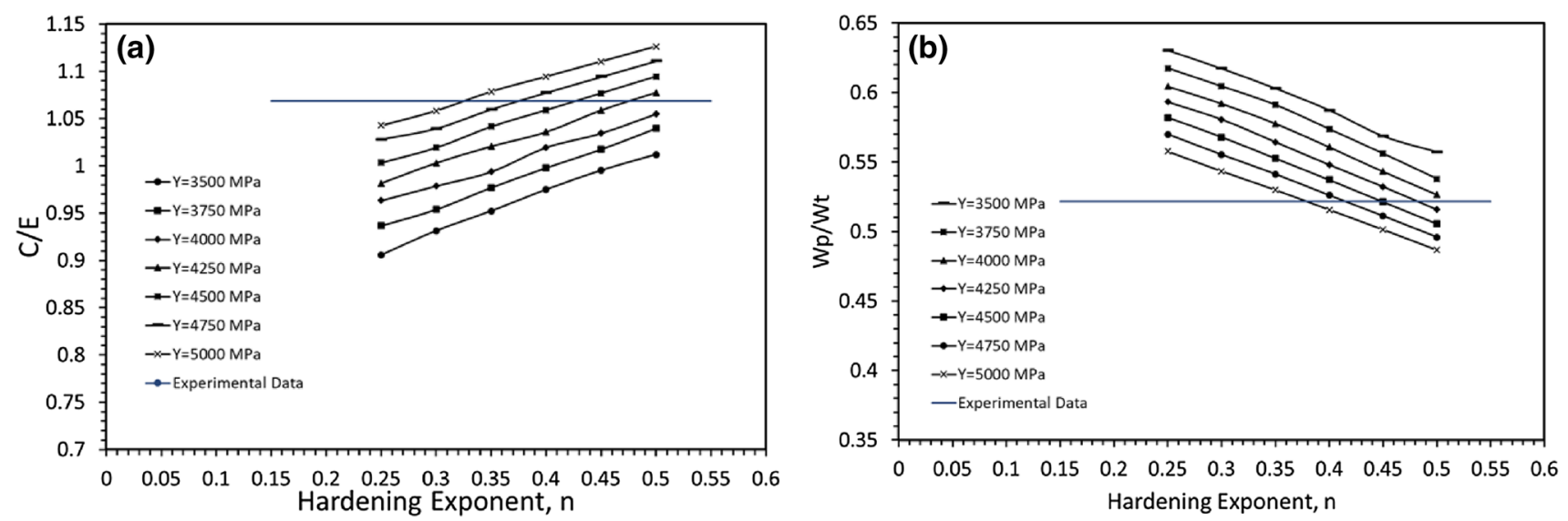

Fig. 11 Variation of dimensionless functions versus hardening exponent, $\mathrm{n}$, for constant yield stress resulted from FEM analysis for AM200 ${ }^{\circledR}$ coating. Experimental data for $\mathbf{a} \mathrm{C} / \mathrm{E}$ and $\mathbf{b} W_{P} / W_{T}$ and the intersections with above lines are demonstrated

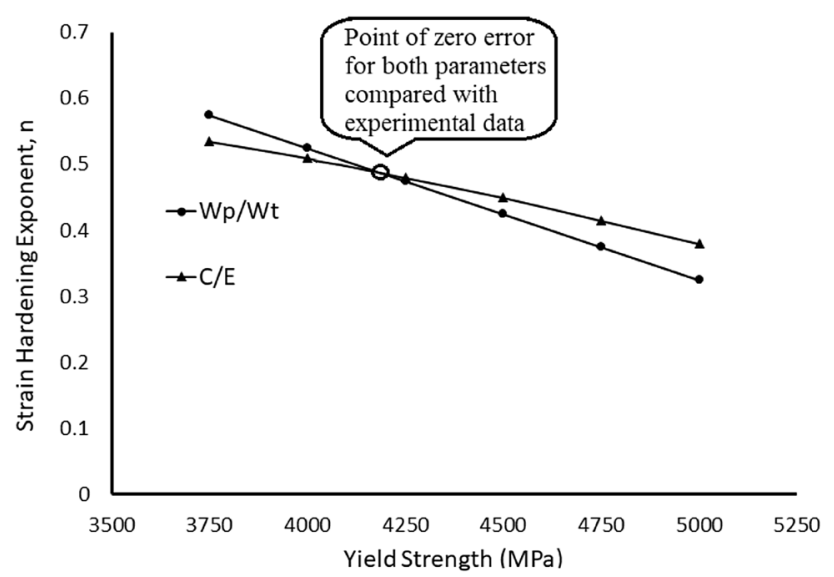

Fig. 12 The Locus of intersection points of experimental and simulation results for $C / E$ and $W_{P} / W_{T}$ parameters. The intersection of the lines represents the plastic characteristics of the coating

such limitations, a new approach is proposed that combines MDAM to the resultant error of both dimensionless parameters. A combination of $\Pi_{\alpha}$ and $\Pi_{\omega}$ errors with respect to experimental reference values is mainly used to search the answer. Such errors that are resulted from Eqs. (13) and (14) can be demonstrated versus the hardening exponent, $\mathrm{n}$, and the yield stress, $\mathrm{Y}$, as shown in Fig. 13a-e.

Figure $13 \mathrm{a}-\mathrm{e}$ clearly shows that the minimums of both parameters do not happen in the same point. This is the main reason that a combination of the errors with minimum values should be defined. For this purpose, the resultant error for each simulation result is calculated according to the relation (17). The exponent, $p$, can significantly affect the resultant errors, which makes it possible to assess the sensibility to this parameter. Moreover, a meaningful relationship is concluded between the variation of each resultant error and its components. Such bilateral relationship can be demonstrated by the curves of Fig. 14a, b. Regardless how much $p$ exponent is, both parameters tend to their minimum values when the value of the resultant error tends to zero. But at the same time the variation trend of both of them do not have the same direction.

For the present case, the resultant errors with $p=1$, 2, 3 and 4 are calculated over all the parameters domain at the vicinity of the probable answer region, i.e., $\sigma_{y} \leq 0.01 E$ and $0.25 \leq n \leq 0.5$. The results which have more apparent difference (i.e., $p=1,2,3$ and $p=4$ ) are shown in Fig. 15a-d as 3D diagrams. Each 3D diagram involves a zone in which the resultant error values are absolutely minimum. In this case, the limited zone between $\sigma_{y}=4000-4500$ and $n=0.45-0.5$ is the rock bottom area. The main finding is that the zone of minimum resultant errors is independent from $p$ although the amount of the error is evidently decreased. To find the characteristics that generate the absolute minimum value, the minimum resultant error values for each certain yield strength is determined. Such values which outline the minimum path of all values in 3D diagram are shown in Fig. 16. It is obviously seen that there is an absolute minimum of resultant error in this minimum path, whatever the value of $p$ exponent is. The best fit point is related to $\sigma_{y} / E=0.0085$ where the resultant error is very close to zero $(0.2 \%)$. Other plastic characteristics can be obtained as: $\sigma_{y}=4280 \mathrm{MPa}, n=0.47$ and $B=33,658 \mathrm{MPa}$. Comparing these results with those of MDAM approach shows very little difference, about $\% 2$ for yield stress and no significant difference for $B$ and $n$. That is what can be considered as one of the best accuracy tolerances in mechanical properties measurement, even in macro-scale. The mentioned properties are almost the same as Inconel 718 alloy which is commonly used in cutting tools (Parida 2018). 
Fig. 13 The Locus of intersection points of experimental and simulation results for $C / E$ and $W_{P} / W_{T}$ parameters. The intersection of the lines represents the plastic characteristics of the coating (a)

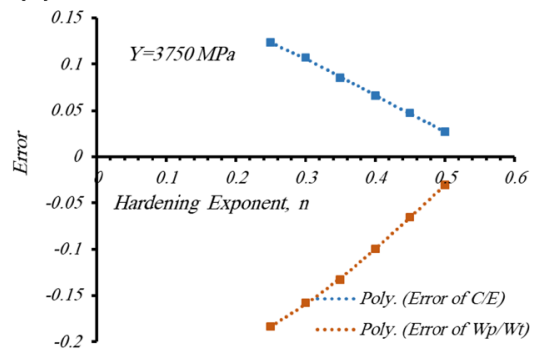

(c)

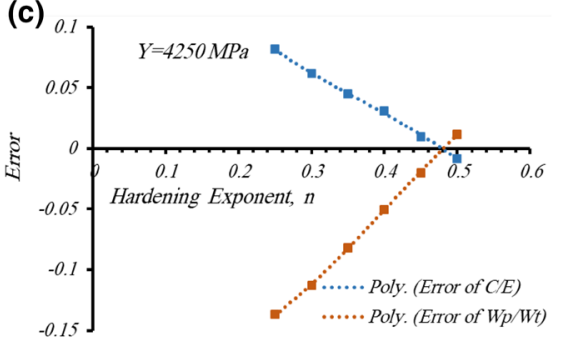

(b)

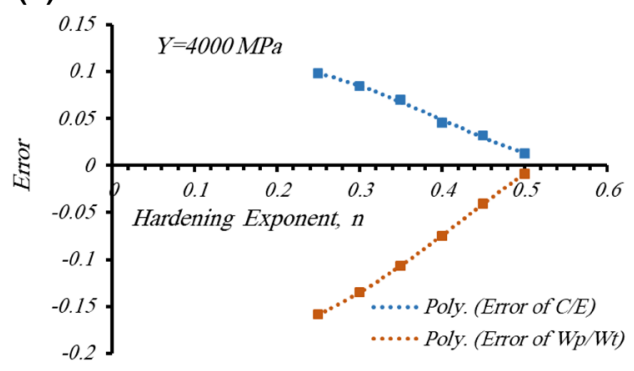

(d)

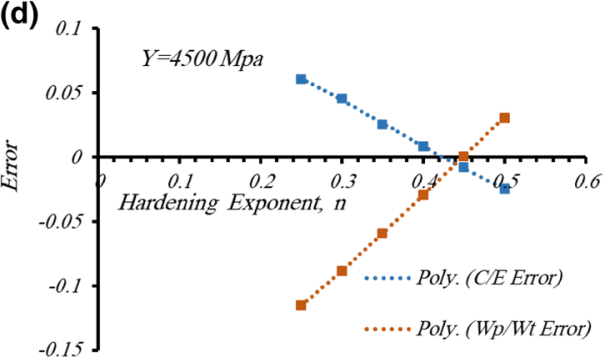

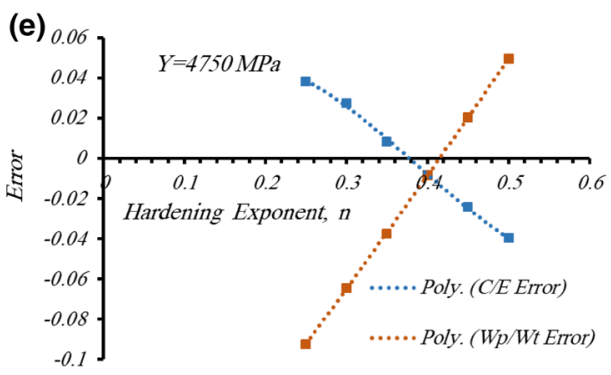
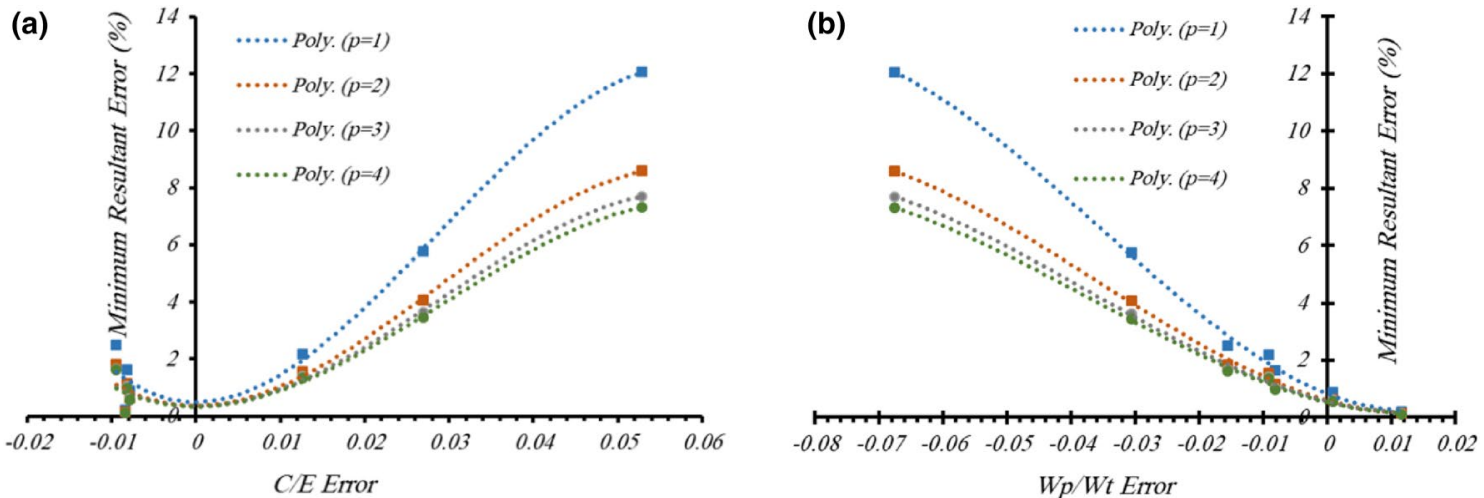

Fig. 14 Variation of the minimum resultant error path versus $\mathbf{a} C / E$ and $\mathbf{b} W_{P} / W_{T}$ for $p=1,2,3$ and 4

The summary of the results illustrates that the infimum resultant error reaches $\% 0.19$, whereas both corresponding individual dimensionless parameters errors are below $\% 0.12$ (as listed in Table 3). This level of error is generally excellent for numerical methods. Diagrams of dimensionless functions in Fig. 13c show this situation, where both functions have nearly zero error values at the same conditions. Comparison of the errors with previous cases reveals that high improvement in approach toward the exact solution is happened. The plastic characteristics as the output results of the combined method are listed in Table 4. Applying the algorithms of MDAM and MREM provides very close results to each other so that the maximum error for yield stress is $1.9 \%$ and for strain hardening coefficient is $0.3 \%$. Strain hardening exponent is obtained the same from both approaches. 


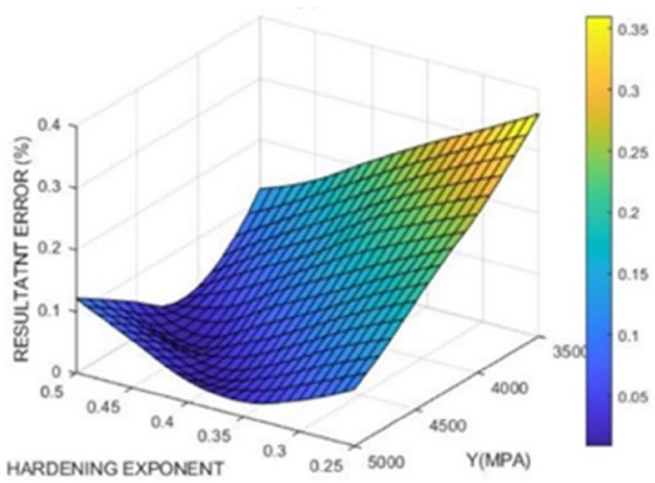

(a) $p=1$

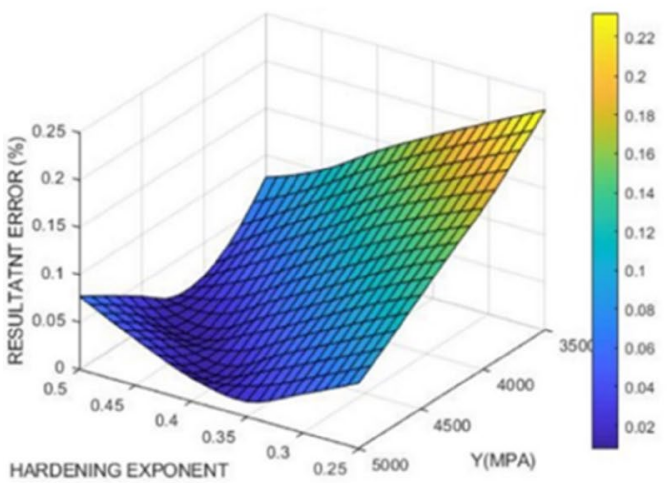

(c) $P=3$

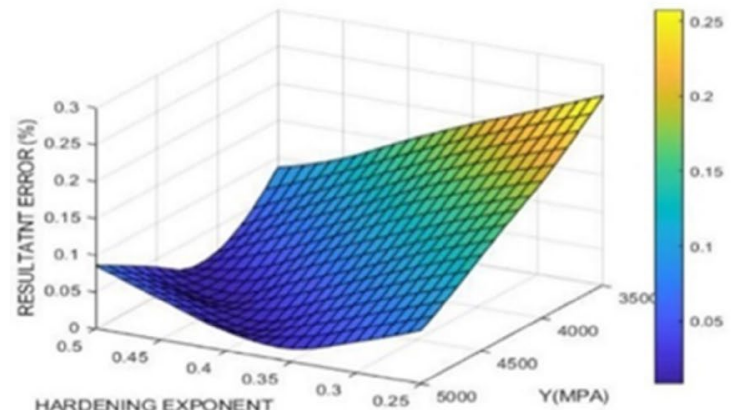

(b) $p=2$

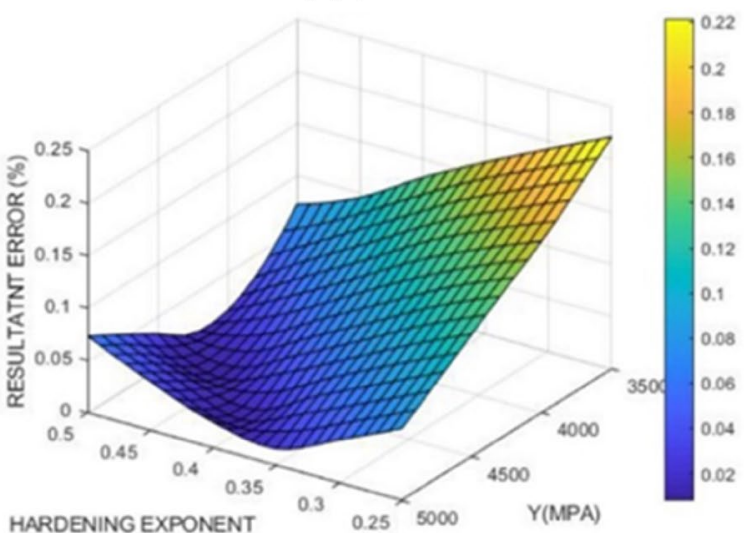

(d) $p=4$

Fig. 15 Variation of the resultant error versus hardening exponent and yield stress for AM200 ${ }^{\circledR}$ coated sample for $p=1,2,3$ and 4

Fig. 16 Minimum path of the resultant errors versus the ratio of yield strength to elastic modulus as a result from full range simulations. The resultant error exponent; $p$ varies from 1 to 4

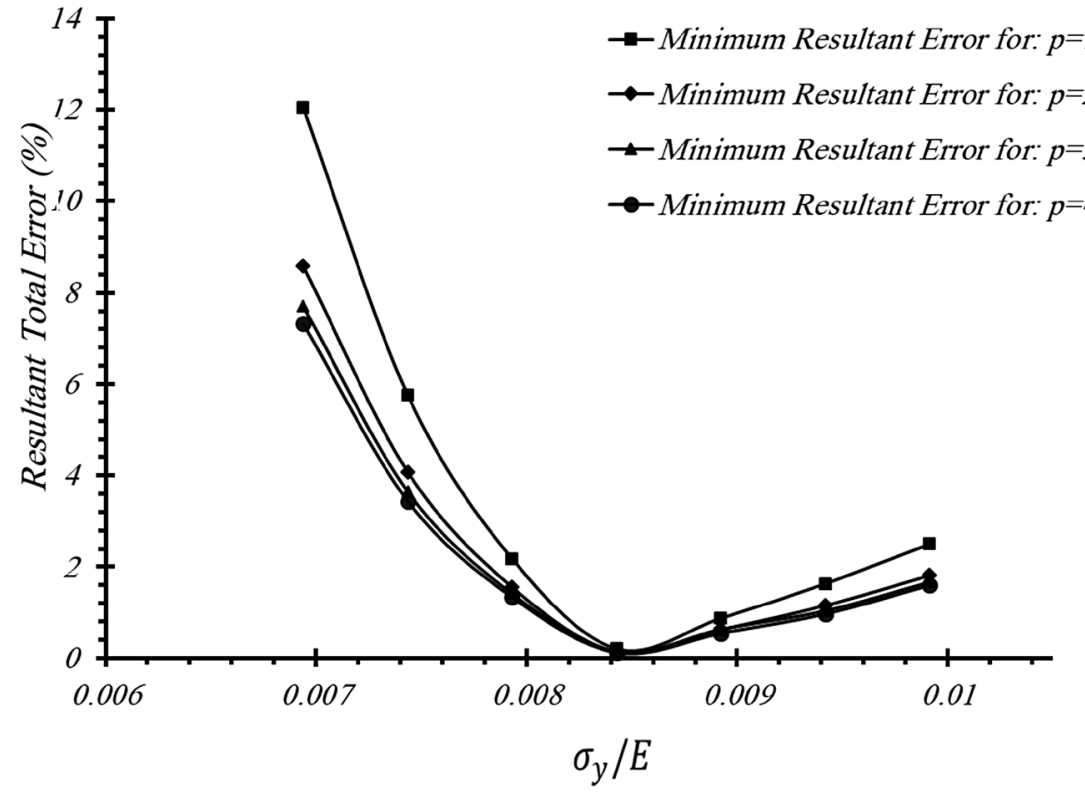


Table 3 Summary of output data of dimensionless parameters errors and resultant error obtained by FEA simulation for AM $200^{\circledR}$, TiN and TiAlN samples

\begin{tabular}{llllll}
\hline Coating & $C / E$ & $\begin{array}{l}C / E \text { error } \\
(\%)\end{array}$ & $W_{P} / W_{T}$ & $\begin{array}{l}W_{P} / W_{T} \text { error } \\
(\%)\end{array}$ & $\begin{array}{l}\text { Resultant } \\
\text { error }(\%)\end{array}$ \\
\hline $\mathrm{AM}^{200}{ }^{\circledR}$ & 1.0689 & -0.084 & 0.52183 & 0.1155 & 0.199 \\
$\mathrm{TiN}^{1}$ & 0.8483 & -2.42 & 0.5467 & -3.34 & 4.1 \\
$\mathrm{TiAlN}^{\mathrm{a}}$ & 0.9517 & -0.47 & 0.6704 & -0.013 & 0.47 \\
\hline
\end{tabular}

${ }^{a}$ According to reference (Bazzaz et al. 2019a)

As a result of comprehensive investigations, the yield stress of AM $200{ }^{\circledR}$ coating is much higher than TiAlN, but it is lower than TiN. Regarding the hardening exponent, $n$, AM200 ${ }^{\circledR}$ has much higher value than others which causes higher hardening strength for this coating, nearly the same as TiN, in spite of the fact that the elastic modulus and yield strength are lower than TiN. This can be clearly observed by comparing the stress-strain diagrams of the different coatings as shown in Fig. 17.

\section{Conclusion}

As the main achievement of this study, the difficulties born by complication of the conventional dimensional analysis were overcome by implying some simplifications included in the MDAM algorithm. Reducing the dimensionless parameters to two parameters was the main modification. Holistically, dimensional analysis methodology was faced with some obstacles in determining the acceptable level of deviations. To solve this problem, another criterion for the error assessment was defined so that the errors of both dimensionless parameters can be combined in one measure. Such criterion can be used as an effective mean to obtain a unique yield stress and hardening exponent. This approach (denoted by MREM) was founded to enable the user to calculate the plastic phase properties by using only one $P-h$ curve coming out of one indenter type. From the previous work of the authors, it was concluded that using the proposed combined algorithm of MDAM and MREM can provide applicable results for bulk materials. In this research work, the same procedure is used for the coating materials and the following results are achieved:
Table 4 Summary of calculation output data of the constitutive equation in elastic and plastic for Ti, AM $200^{\circledR}$, TiN and TiAlN samples

\begin{tabular}{llllll}
\hline Coating & Calculation method & $\begin{array}{l}\text { Young's modu- } \\
\text { lus } E(\mathrm{GPa})\end{array}$ & $\begin{array}{l}\text { Yield Stress } \\
\sigma_{y}(\mathrm{MPa})\end{array}$ & $\begin{array}{l}\text { Hardening } \\
\text { exponent, } n\end{array}$ & $\begin{array}{l}\text { Hardening coef- } \\
\text { ficient } B(\mathrm{MPa})\end{array}$ \\
\hline $\mathrm{Ti}^{\mathrm{a}}$ & MREM & 130 & 487 & 1 & 21,000 \\
$\mathrm{AM} 200^{\circledR}$ & MDAM & 504.4 & 4200 & 0.47 & 33,533 \\
$\mathrm{AM} 200^{\circledR}$ & MREM & 504.4 & 4280 & 0.47 & 33,658 \\
$\mathrm{TiN}^{\mathrm{b}}$ & MREM & 581 & 4800 & 0.4 & 27,600 \\
$\mathrm{TiAlN}^{\mathrm{b}}$ & MREM & 272.2 & 2193 & 0.25 & 6940 \\
\hline
\end{tabular}

${ }^{a}$ According to reference (Bazzaz et al. 2019b)

${ }^{\mathrm{b}}$ According to reference (Bazzaz et al. 2019a)
Fig. 17 Stress-Strain diagram for AM $200^{\circledR}$, TiN and TiAlN according to calculated power law parameters

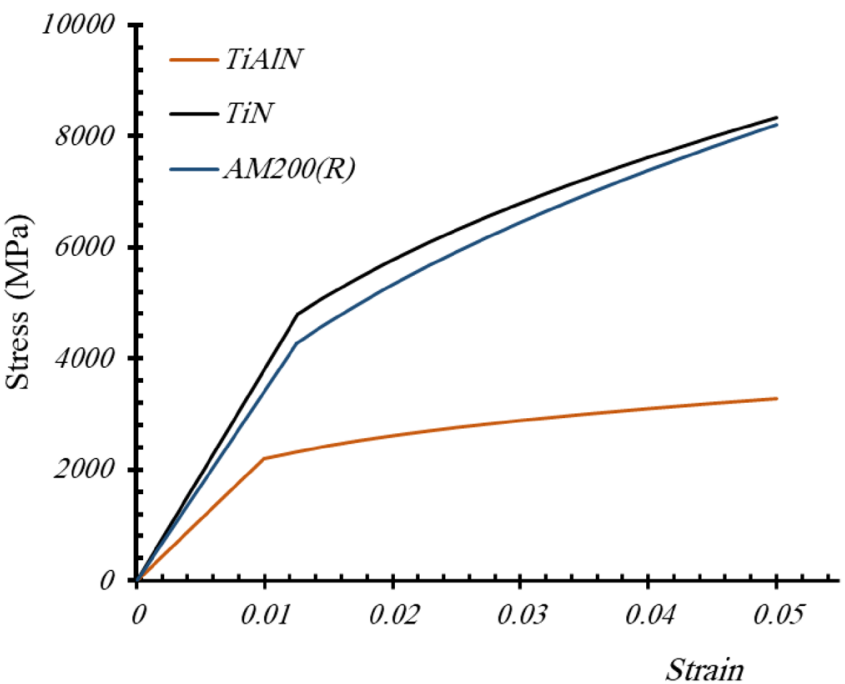


- It is approved that the proposed hybrid method in connection with the modified dimensional analysis provides applicable results when the simulation output is in a good agreement with experimental data.

- Combined MDAM and MREM algorithm is completely applicable for layered materials. The results show less than $\% 2$ error respect to the application of MDAM approach alone.

- The assumptions of the axisymmetric model, rigid indenter, friction between mating faces and round tip indenter are applicable. The results approved that the above-mentioned assumptions are effective in replicating the experimental results.

- The coefficients of Johnson-cook equation are obtained for AM $200^{\circledR}$ successfully. The same coefficients were previously extracted and verified for TiN and TiAlN by the authors. Comparison of the results figures out that the plastic strength of $\mathrm{AM} 200^{\circledR}$ is much higher than TiAlN, but it is a little lower than TiN. This result is in good agreement with the information about the specification of these types of coatings.

- Sensibility investigation of the results to different exponential combination of the errors reveals that the answer is independent from the rank of exponent, $p$, but the level of resultant error is significantly affected. Therefore, assuming $p=2$ provides acceptable results with less than $\% 0.2$ resultant error.

Open Access This article is licensed under a Creative Commons Attribution 4.0 International License, which permits use, sharing, adaptation, distribution and reproduction in any medium or format, as long as you give appropriate credit to the original author(s) and the source, provide a link to the Creative Commons licence, and indicate if changes were made. The images or other third party material in this article are included in the article's Creative Commons licence, unless indicated otherwise in a credit line to the material. If material is not included in the article's Creative Commons licence and your intended use is not permitted by statutory regulation or exceeds the permitted use, you will need to obtain permission directly from the copyright holder. To view a copy of this licence, visit http://creativecommons.org/licenses/by/4.0/.

\section{References}

ABAQUS 6.14 (2014) Analysis User's Guide, Dassault Systems Allied Machine \& Engineering Co. (2018) Hole making Solutions for Today's Manufacturing

American Society for Testing and Materials (2003) ASTM E92. Standard Test Method for Vickers Hardness of Metallic Materials, 82

Antunes J, Menezes L, Fernandes J (2006) Three-dimensional numerical simulation of Vickers indentation tests. Int J Solids Struct 43:784-806

Antunes J, Fernandes J, Menezes L, Chaparro B (2007) A new approach for reverse analyses in depth-sensing indentation using numerical simulation. Acta Mater 55:69-81
ASM Handbook Committee (1989) ASM Handbook: Volume 16: Machining. 16:51-59

Bazzaz E, Darvizeh A, Alitavoli M, Tooski YM (2019a) Investigation of the effect of high rate surface treatment on mechanical properties of titanium base coatings by experimental and numerical analysis. University of Guilan, Department of Mechanical Engineering, Rasht

Bazzaz E, Darvizeh A, Alitavoli M \& Yarmohammad Tooski M (2019b) New hybrid approach in obtaining plastic properties by nanoindentation, finite element method and modified dimensional analysis. Accepted Manuscript, J Sci Technol Compos

Bobzin K, Lugscheider E, Maes M, Bolz S (2007) Grain size evaluation of pulsed TiAlN nanocomposite coatings for cutting tools. Thin Solid Films 515:3681-3684

Cao YP, Lu J (2004) A new method to extract the plastic properties of metal materials from an instrumented spherical indentation loading curve. Acta Mater 52:4023-4032

Challacoop N, Dao M, Suresh S (2003) Depth-sensing instrumented indentation with dual sharp Indenters. Acta Mater 51:3713-3729

Cheng YT, Cheng CM (1999) Can stress-strain relationships be obtained from indentation curves using conical and pyramidal indenters? J Mater Res 14:3493-3496

Cheng YT, Cheng CM (2004) Scaling, dimensional analysis and indentation measurements. Mater Sci Eng 44:91-149

Cheng YT, Tay B, Lau S (2002) Electrical properties of TiN films deposited by filtered cathodic vacuum arc. J Vac Sci Technol, B 20:2000

Dao $M$ et al (2001) Computational modeling of the forward and reverse problems in instrumented sharp indentation. Acta Mater 49:3899-3918

Doerner M, Nix W (1986) A method for interpreting the data from depth-sensing indentation instruments. J Mater Res 1:601-609

dos Santos J et al (2007) Tribological evaluation of TiN and TiAlN coated PM-HSS gear cutter when machining $19 \mathrm{MnCr} 5$ steel. Int J Adv Manuf Technol 31:629-637

Duodu EA et al (2016) Damage induced by high-velocity impact on composite structures using finite element simulation. Iran J Sci Technol Trans Mech Eng. https://doi.org/10.1007/s4099 7-016-0047-z

Fang E, Jian SR, Chuu DS (2004) Nanomechanical properties of TiC, $\mathrm{TiN}$ and TiCN thin films using scanning probe microscopy and nanoindentation. Appl Surf Sci 228:365-372

Fischer-Cripps A (2011) Nanoindentation, Chapter 6: scaling relationships in nanoindentation, 3rd edn. Springer, New York

Giannakopoulos A, Suresh S (1999) Determination of elastoplastic properties by instrumented sharp indentation. Scripta Mater 40:1191-1198

Giannakopoulos A, Larsson P, Vester R (1994) Analysis of Vikers indentation. Int J Solids Struct 31:2679-2708

Gupta AK et al (2015) Evaluation of elasto-plastic properties of ITO film using combined nanoindentation and finite element approach. Ceram Int. https://doi.org/10.1016/j.ceramint.2015.09.054

Gupta AK et al (2016) Evaluation of critical depth ratio for soft V2O5 film on hard Si substrate by finite element modeling of experimentally measured nanoindentation response. J Phys D Appl Phys 49:155302. https://doi.org/10.1088/0022-3727/49/15/155302

Heinrich C, Waas A, Wineman A (2009) Determination of material properties using nanoindentation and multiple indenter tips. Int $\mathbf{J}$ Solids Struct 46:364-376

Hong RY, Cheng HC, Hu HC, Chen WH (2016) Characterization of elastic-plastic properties of intermetallic compounds. In: ICEP Proceedings

ISO-14577 (2002) In: Part 2 \& 3. Geneva, Switzerland

Kang J, Becker A, Sun W (2012) determining elasticplastic properties from indentation data obtained from finite element simulations and experimental results. Int J Mech Sci 62:34-46 
Karimzadeh A, Ayatollahi M, Alizadeh M (2014) Finite element simulation of nano-indentation experiment on aluminum 1100. Comput Mater Sci 81:595-600

Kataria S et al (2012) Evaluation of nano-mechanical properties of hard coatings on a soft substrate. Thin Solid Films 522:297-303

Lee J, Lee C, Kim B (2009) Reverse analysis of nano-indentation using different representative strains and residual indentation profiles. Mater Des 30:3395-3404

Li W, Huang C, Yu M, Liao H (2013) Investigation on mechanical property of annealed copper particles and cold sprayed copper coating by a micro-indentation testing. Mater Des 46:219-226

Lichinchi M, Lenardi C, Haupt J, Vitali R (1998) Simulation of Berkovich nanoindentation experiment on thin films using the finite element method. Thin Solid Films 312:240-248

Lin J, Niu XY, Shu XF (2008) Reverse analysis for determining the mechanical properties of zeolite ferrierite crystal. J Nanomater. https://doi.org/10.1155/2008/395738

Ma Z, Zhou Y, Long S, Lu C (2012) An inverse approach for extracting elastic-plastic properties of thin films from small scale sharp indentation. J Mater Sci Technol 28(7):626-635

Malzbender J (2002) Indentation load-displacement, plastic deformation, and energy. J Mater Res 17:502-511

Oliver W, Pharr G (1992) An improved technique for determining hardness and elastic modulus using load and displacement sensing indentation experiments. J Mater Res 7:1564-1583

Parida A (2018) Analysis of chip geometry in hot machining of Inconel 718 alloy. Iran J Sci Technol Trans Mech Eng. https:// doi.org/10.1007/s40997-018-0146-0

Patel D, Kalidindi S (2016) Correlation of spherical nanoindentation stress-strain curves to simple compression stress-strain curves for elastic-plastic isotropic materials using finite element models. Acta Mater 112:295-302

Piscanec S et al (2004) Bioactivity of TiN-coated titanium implants. Acta Mater 52:1237-1245
Porwal D et al (2016) Simulation of nanoindentation experiment on RF magnetron sputtered nanocolumnar V2O5 film using finite element method. Mater. Res. Express 3:076407. https://doi. org/10.1088/2053-1591/3/7/076407

Sakharova N, Fernandes J, Antunes J, Olivier W (2009) Comparison between Berkovich, Vickers and conical indentation tests: a three-dimensional numerical simulation study. Int J Solids Struct 46:1095-1104

Stauss S et al (2003) Determining the stress-strain behaviour of small devices by nanoindentation in combination with inverse methods. Microelectron Eng 67-68:818-825

Stopel M, Skibicki D (2016) Determination of Johnson-Cook model constants by measurement of strain rate by optical method. 1780 , 060003

Sun Y et al (2015) Nanomechanical properties of TiCN and TiCN/ Ti coatings on Ti prepared by Filtered Arc deposition. Mater Sci Eng A 625:56-64

Suresh S, Giannakopoulos A, Alcala J (1997) Spherical indentation of compositionally graded materials: theory and experiments. Acta Mater 45:1307-1321

Vaidyanathan R, Dao M, Ravichandran G, Suresh S (2001) Study of mechanical deformation in bulk metallic glass through instrumented indentation. Acta Mater 49:3781-3789. https://doi. org/10.1016/S1359-6454(01)00263-4

Wang J et al (2010) Identification of elastic parameters of transversely isotropic thin films by combining nanoindentation and FEM analysis. Comput Mater Sci 49:378-385

Wen W, Becker A, Sun W (2017) Determination of material properties of thin films and coatings using indentation tests: a review. J Mater Sci 52:12553

Zhaohui S, Suresh S (2003) Elastic-plastic characterization of thin films using nanoindentation technique. Thin Solid Films 437:176-182 